\title{
Impfprävention - das unverzichtbare Zusatzbrot
}

\author{
Das Honorarvolumen für die einzelne Praxis wird ab 1. Juli rigoros begrenzt. \\ Der Vertragsarzt muss sich also schleunigst auf Bereiche fokussieren, die noch \\ Umsatzsteigerungen möglich machen. Prävention ist angesagt und hier im \\ Besonderen die Impfungen. Diese sind ohne Begrenzung und extrabudgetär.
}

- Mit konsequentem Impfmanagement kann eine durchschnittliche Hausarztpraxis pro Quartal einen extrabudgetären zusätzlichen Umsatz von 1000 Euro erzielen.

Mit der Präventionsleistung Impfen gewinnen kleine und große Patienten, aber auch der Hausarzt. Junge und alte Patienten gewinnen an Lebensqualität und Gesundheit, denn keine präventive Maßnahme ist so effektiv wie Impfen.

Seit dem 1. April 2007 sind Schutzimpfungen nicht mehr freiwillige Satzungsleistungen der Krankenkassen, sondern Pflichtleistungen der GKV. Der Gemeinsame Bundesausschuss (G-BA) hatte die Aufgabe, eine entsprechende Richtlinie, die „Schutzimpfungsrichtlinie“" $\mathrm{SiR}$ ), zu erstellen. Der G-BA übernahm im Rahmen der SiR die Empfehlungen der Ständigen Impfkommission am Robert Koch-Institut (STIKO). Damit sind diese STIKOEmpfehlungen für alle am Gesundheits-

\section{Seminarankündigung EBM 2010}

Unser Gebührenordnungsexperte Helmut Walbert macht Sie rechtzeitig fit mit Strategien zu Qualitätsbezogenen Zusatzvolumen (OZV), RLV, Budgets:

Orte und Termine:

Hannover: 30. Juni 2010

Hamburg: 2. Juli 2010

Nürnberg: 7. Juli 2010

München: 10. Juli 2010

Nähere Infos unter:

Tel.: 09312998594

Fax: 093125090601

E-Mail:w@lbert.info

Website: www.walbert-helmut.de wesen Beteiligten verbindlich. Allerdings können und dürfen einzelne Krankenkassen über diese Pflichtleistung der GKV hinaus als sogenannte Satzungsleistung auch weitere Impfungen übernehmen und bezahlen. So werden beispielsweise Reiseimpfungen zurückerstattet oder von der STIKO empfohlene Impfungen über die vorgeschriebene Altersgrenze hinaus übernommen.

\section{Länderspezifische Besonderheiten beachten}

Dennoch werden immer wieder länderspezifische Besonderheiten vereinbart. So konnte in der letzten Wintersaison in Bayern über die Indikationsgruppe hinaus jeder, der es wollte, zulasten der GKV gegen Grippe geimpft werden. Hier gilt es, sich rechtzeitig zu Beginn einer jeden Saison bei der örtlichen Kassenärztlichen Vereinigung (KV) zu informieren. Nur so können Umsatzverluste vermieden werden.

\section{Information ist der Schlüssel zum Erfolg}

Information ist im Impfmanagement das $\mathrm{A}$ und $\mathrm{O}$, um eine möglichst hohe Quote an geimpften Patienten zu erreichen. Die von der STIKO empfohlenen Impfungen müssen dem gesamten Praxisteam präsent sein. An den wichtigsten Stellen in der Praxis sollte ein aktuelles Exemplar des offiziellen Impfkalenders der STIKO entweder hängen oder griffbereit sein. So können eventuell Zweifel sofort beseitigt werden.
Die niedergelassenen Haus- und Kinderärzte wurden auf der 1 . Nationalen Impfkonferenz getadelt, weil die Impfquote in weiten Bereichen zu wünschen übrig lässt.

So zeigt die verfügbare Datenlage zur Durchimmunisierung von Jugendlichen bundesweit, länderbezogen und auch kommunal erhebliche Impfdefizite bei älteren Schülern: Die Impfquoten liegen für die Auffrischimpfung gegen Tetanus und Diphtherie bundesweit unter $40 \%$ und für die Auffrischimpfung Pertussis sogar noch niedriger. Die nachzuholende Hepatitis-B-Grundimmunisierung liegt bei knapp $60 \%$ und bei der zweiten MMR unter 75\%. Regional spezifisch führten zu niedrige MMRImpfquoten zu Masernausbrüchen.

\section{Aktives Impfmanagement gefragt}

Hier ist also für die Haus- und Kinderarzt-Praxis ein lohnenswertes Betätigungsfeld. Viele Patienten begrüßen ein aktives Impfmanagement einschließlich eines Recallprogramms.

Ab sofort sollte bei jedem Arzt-Patienten-Kontakt der Impfstatus angesprochen werden, die Impfbücher überprüft und damit der Impfstatus in der elektronischen Karteikarte in der Praxis auf dem neuesten Stand sein. Die fehlenden Impfungen sollten zu einer entsprechenden Markierung in der EDV führen, die mit Aufrufen des Patienten sofort ins Auge fällt. Auch die nächstfällige Auffrischimpfung sollte entsprechend dokumentiert sein und mit Aufruf des Patienten in der EDV sichtbar sein. Dies auch dann, wenn es noch einige Jahre Zeit haben sollte. Auf diese Weise wird jede fällige Impfung rechtzeitig erinnert und es geht kein Umsatz verloren!

H. WALBERT a 